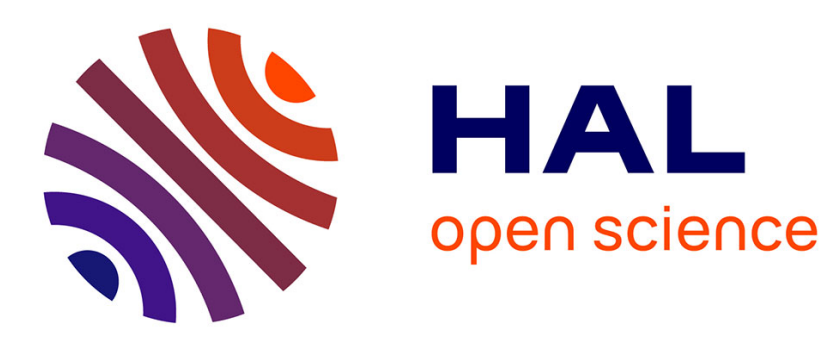

\title{
Canonical Decomposition of Basic Belief Assignment for Decision-Making Support
}

\author{
Jean Dezert, Florentin Smarandache
}

\section{To cite this version:}

Jean Dezert, Florentin Smarandache. Canonical Decomposition of Basic Belief Assignment for Decision-Making Support. 7th International Conference MDIS 2020, Oct 2020, Sibiu, Romania. hal03105976

\section{HAL Id: hal-03105976 https://hal.science/hal-03105976}

Submitted on 11 Jan 2021

HAL is a multi-disciplinary open access archive for the deposit and dissemination of scientific research documents, whether they are published or not. The documents may come from teaching and research institutions in France or abroad, or from public or private research centers.
L'archive ouverte pluridisciplinaire HAL, est destinée au dépôt et à la diffusion de documents scientifiques de niveau recherche, publiés ou non, émanant des établissements d'enseignement et de recherche français ou étrangers, des laboratoires publics ou privés. 


\title{
Canonical Decomposition of Basic Belief Assignment for Decision-Making Support
}

\author{
Jean Dezert ${ }^{1}$ and Florentin Smarandache ${ }^{2}$ \\ 1 The French Aerospace Lab, Palaiseau, France. \\ jean.dezert@onera.fr \\ 2 Dept. of Mathematics, Univ. of New Mexico, Gallup, NM, USA. \\ smarand@unm.edu
}

\begin{abstract}
We present a new methodology for decision-making support based on belief functions thanks to a new theoretical canonical decomposition of dichotomous basic belief assignments (BBAs) that has been developed recently. This decomposition based on proportional conflict redistribution rule no 5 (PCR5) always exists and is unique. This new PCR5-based decomposition method circumvents the exponential complexity of the direct fusion of BBAs with PCR5 rule and it allows to fuse quickly many sources of evidences. The method we propose in this paper provides both a decision and an estimation of the quality of the decision made, which is appealing for decision-making support systems.
\end{abstract}

Keywords: Decision-making · Belief functions · PCR5.

\section{Introduction}

This paper deals with the decision-making support problem from many sources of evidence characterized by belief functions (BF) defined over a same frame of discernment. Belief functions introduced by Shafer [1] are appealing to model epistemic uncertainty. They are well-known and used in the artificial intelligence community to fuse uncertain information and to make a decision. However, many debates in scientific community started with Zadeh's criticism $[2,3]$ - see additional references in [4] - have bloomed on the validity of Dempster's rule of combination and its counter-intuitive behavior (not only in high conflicting situations, but also in low conflicting situations as well). That is why many rules of combination have been developed by different researchers [5] (Vol. 2) over the last decades. In this work we consider only the rule based on the proportional conflict redistribution principle no 5 (PCR5 rule) to combine basic belief assignments (BBAs). This choice is motived not only by its conflict redistribution principle, but also by its ability to generate a unique canonical decomposition of any dichotomous BBA that will be convenient for decision-making from many sources of evidence.

This paper is organized as follows. After a brief recall of basics of belief functions in section 2, we present succinctly the canonical decomposition of a 
(dichotomous) BBA in section 3 based on [6]. Then we propose a new decisionmaking support methodology that exploits this canonical decomposition in section 4 for working in a general framework with many (non dichotomous) sources of evidences, with basic illustrative examples. Conclusions are given in section 5 .

\section{Basics of belief functions}

\subsection{Definitions}

The answer ${ }^{3}$ of the problem under concern is supposed to belong to a given finite discrete frame of discernment (FoD) $\Theta=\left\{\theta_{1}, \theta_{2}, \ldots, \theta_{n}\right\}$, with $n>1$. All elements of $\Theta$ are mutually exclusive ${ }^{4}$. The set of all subsets of $\Theta$ (including empty set $\emptyset$ and $\Theta$ ) is the power-set of $\Theta$ denoted by $2^{\Theta}$. A Basic Belief Assignment (BBA) given by a source of evidence is defined [1] as $m(\cdot): 2^{\Theta} \rightarrow[0,1]$ satisfying $m(\emptyset)=0$ and $\sum_{A \in 2{ }^{\Theta}} m(A)=1$. The quantity $m(A)$ is the mass of belief of $A$. Belief and plausibility functions are respectively defined from $m(\cdot)$ by

$$
\operatorname{Bel}(A)=\sum_{B \in 2^{\Theta} \mid B \subseteq A} m(B)
$$

and

$$
P l(A)=\sum_{B \in 2^{\Theta} \mid A \cap B \neq \emptyset} m(B)=1-\operatorname{Bel}(\bar{A}) .
$$

where $\bar{A}$ is the complement of $A$ in $\Theta$.

$\operatorname{Bel}(A)$ and $\operatorname{Pl}(A)$ are usually interpreted respectively as lower and upper bounds of an unknown (subjective) probability measure $P(A) . A$ is called a Focal Element $(\mathrm{FE})$ of $m(\cdot)$ if $m(A)>0$. When all focal elements are singletons then $m(\cdot)$ is called a Bayesian $B B A[1]$ and its corresponding $B e l(\cdot)$ function is equal to $P l(\cdot)$ and they are homogeneous to a (subjective) probability measure $P(\cdot)$. The vacuous BBA (VBBA for short) representing a totally ignorant source is defined $\operatorname{as}^{5} m_{v}(\Theta)=1$. A dogmatic BBA is a BBA such that $m(\Theta)=0$. If $m(\Theta)>0$ the BBA $m(\cdot)$ is nondogmatic. A simple BBA is a BBA that has at most two focal sets and one of them is $\Theta$. A FoD is a dichotomous FoD if it has only two elements, say $\Theta=\{A, \bar{A}\}$ with $A \neq \emptyset$ and $A \neq \Theta$. A dichotomous BBA is a BBA defined over a dichotomous FoD.

\subsection{PCR5 rule of combination}

The combination of distinct sources of evidence characterized by their BBAs is done by Dempster's rule of combination in Shafer's mathematical theory of

\footnotetext{
${ }^{3}$ I.e. the solution, or the decision to take.

${ }^{4}$ This is so-called Shafer's model of FoD [5].

${ }^{5}$ The complete ignorance is denoted $\Theta$ in Shafer's book [1].
} 
evidence [1]. The justification and behavior of Dempster's rule (corresponding to the normalized conjunctive rule) have been disputed from many counterexamples involving high and low conflicting sources (from both theoretical and practical standpoints) as reported in [4]. Many alternatives to Dempster's rule are now available [5], Vol. 2. Among them, we consider in the sequel the PCR5 rule which transfers the conflicting mass only to the elements involved in the conflict and proportionally to their individual masses, so that a more sophisticate and precise distribution is done with the PCR5 fusion process. The PCR5 rule is presented in details (with justification and examples) in [5], Vol. 2 and Vol. 3. We only briefly recall for convenience its formula for the fusion of two BBAs, which is symbolically noted as $m_{P C R 5}=P C R 5\left(m_{1}, m_{2}\right)$, where $P C R 5(\cdot, \cdot)$ represents the PCR5 fusion rule for two BBAs. With this PCR5 rule, one has $m_{P C R 5}(\emptyset)=$ 0 , and $\forall X \in 2^{\Theta} \backslash\{\emptyset\}$

$$
m_{P C R 5}(X)=m_{C o n j}(X)+\sum_{\substack{X_{2} \in 2^{\Theta} \\ X_{2} \cap X=\emptyset}}\left[\frac{m_{1}(X)^{2} m_{2}\left(X_{2}\right)}{m_{1}(X)+m_{2}\left(X_{2}\right)}+\frac{m_{2}(X)^{2} m_{1}\left(X_{2}\right)}{m_{2}(X)+m_{1}\left(X_{2}\right)}\right]
$$

where $m_{C o n j}(X)=\sum_{\substack{X_{1}, X_{2} \in 2^{\Theta} \\ X_{1} \cap X_{2}=X}} m_{1}\left(X_{1}\right) m_{2}\left(X_{2}\right)$ is the conjunctive rule, and where all denominators in (3) are different from zero. If a denominator is zero, that fraction is discarded. Extension of PCR5 for combining qualitative BBA's can be found in [5], Vols. $2 \& 3$. All propositions/sets are in a canonical form. A variant of PCR5, called PCR6 has been proposed by Martin and Osswald in [5], Vol. 2, for combining $s>2$ sources. The general formulas for PCR5 and PCR6 rules are also given in [5], Vol. 2. PCR6 coincides with PCR5 when one combines two sources. The difference between PCR5 and PCR6 lies in the way the proportional conflict redistribution is done as soon as three (or more) sources are involved in the fusion.

\section{Canonical decomposition of a dichotomous BBA}

Because the canonical decomposition of a dichotomous BBA has been presented in details in [6], we only make a succinct presentation here. A FoD is a dichotomous $\mathrm{FoD}$ if it is made of only two elements, say $\Theta=\{A, \bar{A}\}$ with $A \cup \bar{A}=\Theta$ and $A \cap \bar{A}=\emptyset . A$ is different from $\Theta$ and from Empty-Set because we want to work with informative FoD. A dichotomous BBA $m(\cdot): 2^{\Theta} \rightarrow[0,1]$ has the general form

$$
m(A)=a, \quad m(\bar{A})=b, \quad m(A \cup \bar{A})=1-a-b
$$

with $a, b \in[0,1]$ and $a+b \leq 1$.

The canonical decomposition problem consists in finding the two following simpler BBAs $m_{p}$ and $m_{c}$ of the form

$$
m_{p}(A)=x, \quad m_{p}(A \cup \bar{A})=1-x
$$




$$
m_{c}(\bar{A})=y, \quad m_{c}(A \cup \bar{A})=1-y
$$

with $(x, y) \in[0,1] \times[0,1]$, such that $m=$ Fusion $\left(m_{p}, m_{c}\right)$, for a chosen rule of combination denoted by Fusion $(\cdot, \cdot)$. The simple BBA $m_{p}(\cdot)$ is called the pro- $B B A$ (or pro-evidence) of $A$, and the simple BBA $m_{c}(\cdot)$ the contra-BBA (or contra-evidence) of $A$. The BBA $m_{p}(\cdot)$ is interpreted as a source of evidence providing an uncertain evidence in favor of $A$, whereas $m_{c}(\cdot)$ is interpreted as a source of evidence providing an uncertain contrary evidence about $A$. In [6], we proved that this decomposition always exists and is unique if we use the PCR5 fusion rule. In the vacuous BBA case when $a=0$ and $b=0$, the BBA $m(\cdot)$ can be interpreted as the PCR5 fusion of two degenerate pro- and contraevidences BBAs $m_{p}(\cdot)$ and $m_{c}(\cdot)$ which coincide with the vacuous BBA with $x=0$ and $y=0$. Hence any (Bayesian, or non Bayesian) dichotomous BBA $m(\cdot)$ can be always interpreted as the result of the PCR5 fusion of these two (pros and cons) aspects of evidence about $A$. It is worth noting that this type of canonical decomposition is different of Smets' canonical decomposition problem [7] which needs to work with generalized simple BBA which are not stricto sensu valid BBAs as defined by Shafer [1].

For the case of dichotomous dogmatic BBA, the expression of solutions $x$ and $y$ of canonical decomposition are as follows $[6]$ :

- if $a=b$ and $a+b=1$ then $a=b=0.5$ and $x=y=1$;

- if $a<b$ then $x<y$, and we have $y=1$ and $x=\frac{a+\sqrt{a^{2}+4 a}}{2}$;

- if $a>b$ then $x>y$, and we have $x=1$ and $y=\frac{b+\sqrt{b^{2}+4 b}}{2}$.

For the case of dichotomous non-dogmatic BBA, the expression of solutions $x$ and $y$ of the canonical decomposition do not have simple analytical expression because one has to find $x$ and $y$ solutions of the system

$$
\begin{aligned}
& a=x(1-y)+\frac{x^{2} y}{x+y}=\frac{x^{2}+x y-x y^{2}}{x+y} \\
& b=(1-x) y+\frac{x y^{2}}{x+y}=\frac{y^{2}+x y-x^{2} y}{x+y}
\end{aligned}
$$

under the constraints $(a, b) \in[0,1]^{2}$, and $0<a+b<1$. In fact, we have proved in [6] that $x \in[a, a+b] \subset[0,1]$ and $y \in[b, a+b] \subset[0,1]$, but the explicit expression of $x$ and $y$ are very complicated to obtain analytically (even with modern symbolic computing systems like Mathematica ${ }^{\mathrm{TM}}$, or Maple ${ }^{\mathrm{TM}}$ ) because after algebraic calculation, and for $x \neq 1$, one has to solve the following quartic equation which has at most four real solutions with only a valid one in $[a, a+b]$

$$
x^{4}+(-a-2) x^{3}+(2 a+b) x^{2}+\left(a+b-a b-b^{2}\right) x+\left(-a^{2}-a b\right)=0
$$

and then compute $y$ by $y=(a+b-x)(1-x)$.

Once the numerical values are committed to $a$ and to $b$ the numerical (approximate) solutions $x$ and then $y$ can be easily obtained by a standard numerical 
solver. For instance, with Matlab ${ }^{\mathrm{TM}}$ we can use the fsolve command, and this is what we use to make the canonical decomposition of dichotomous non-dogmatic BBA.

\subsection{Canonical decompositions from other well-known rules}

In [6] we did prove that this type of canonical decomposition cannot be obtained by the conjunctive rule only, because if $m_{p}$ and $m_{c}$ exist and if $x>0$ and $y>0$ then $m_{C o n j}(\emptyset)=x \cdot y>0$ which means that $m=\operatorname{Conj}\left(m_{p}, m_{c}\right)$ is not a proper BBA as defined by Shafer's. If we use the disjunctive rule of combination we will always obtain the vacuous BBA as the result ${ }^{6}$ of $\operatorname{Disj}\left(m_{p}, m_{c}\right)$ because $m_{p}(A) m_{c}(\bar{A}), m_{p}(A) m_{c}(A \cup \bar{A}), m_{p}(A \cup \bar{A}) m_{c}(\bar{A})$ and $m_{p}(A \cup \bar{A}) m_{c}(A \cup \bar{A})$ will all be committed to the uncertainty $A \cup \bar{A}$. So for any choice of $m_{p}$ and $m_{c}$ we always get same result (the vacuous BBA) when using the disjunctive rule making the canonical decomposition of non vacuous dichotomous BBA $m$ just impossible. Due to the particular simple form of BBAs $m_{p}(\cdot)$ and $m_{c}(\cdot)$, Yager's rule [8] and Dubois-Prade rule [9] coincide, and we have to search $x$ and $y$ in $[0,1]$ such that $m(A)=a=x(1-y)$ and $m(\bar{A})=b=(1-x) y$. Assuming $^{7} y<1$, one gets from the first equation $x=a /(1-y)$. By replacing $x$ by its expression in the second equation $y-x y=b$ we have to find $y$ in $[0,1)$ such that (after basic algebraic simplifications) $y^{2}+(a-b-1) y+b=0$. This 2 nd order equation admits one or two real solutions $y_{1}$ and $y_{2}$ if and only if the discriminant is null or positive respectively, that is if $(a-b-1)^{2}-4 b \geq 0$. However this discriminant can become negative depending on the values of $a$ and $b$. For instance, for $a=0.3$ and $b=0.6$, we have $(a-b-1)^{2}-4 b=-0.71$ which means that there is no real solution for the equation $y^{2}-1.3 \cdot y+0.6=0$. Therefore, in general (that is for all possible values $a$ and $b$ of the BBA $m$ ), the canonical decomposition of the BBA $m(\cdot)$ cannot be obtained from Yager's and Dubois \& Prade rules of combination. If we use the averaging rule, we are searching $x$ and $y$ in $[0,1]$ such that $m(A)=a=(x+0) / 2$ and $m(\bar{A})=b=(0+y) / 2$, which means that $x=2 a$ and $y=2 b$ with $x$ and $y$ in $[0,1]$. So, if $a>0.5$ or $b>0.5$ the canonical decomposition is impossible to make with the averaging rule of combination. Therefore, in general, the averaging rule is not able to provide a canonical decomposition of the BBA $m(\cdot)$.

If we consider the canonical decomposition of a dichotomous non-dogmatic BBA $(a+b<1)$ using Dempster's rule of combination [1], denoted $D S\left(m_{p}, m_{c}\right)$, we have to obtain $x$ and $y$ in $[0,1]$ such that $^{8} x y \neq 1$ and

\footnotetext{
${ }^{6} \operatorname{Disj}\left(m_{p}, m_{c}\right)$ denotes symbolically the disjunctive fusion of $m_{p}$ with $m_{c}$.

7 Taking $y=1$ would means that $x(1-y)=0$ but $m(A)=a$ with $a \neq 0$ in general, so the choice of $y=1$ is not possible.

8 The third equality $m(A \cup \bar{A})=1-a-b=\frac{(1-x)(1-y)}{1-x y}$ being redundant with (10) and (11) is useless.
} 


$$
\begin{aligned}
& m(A)=a=\frac{x(1-y)}{1-x y} \\
& m(\bar{A})=b=\frac{y(1-x)}{1-x y}
\end{aligned}
$$

with the constraints $0<x<1$ and $0<y<1$. Therefore,

$$
x=\frac{a}{1-y+a y}, \quad y \neq \frac{1}{1-a}
$$

and we solve the equation $y-x y+b x y=b$ with $x$ expressed as function of $y$ as above. We get the equation for $a \neq 1$

$$
(a-1) y^{2}+(1+b-a) y-b=0
$$

whose two solutions are $y_{1}=b /(1-a)$ and $y_{2}=1$ - see [6] for details.

For the case $a \neq 1$, the second "solution" $y_{2}=1$ implies $x=\frac{a}{1-y_{2}+a y_{2}}=$ $\frac{a}{a}=1$ which is not an acceptable solution ${ }^{9}$ because one must have $x y \neq 1$. The solution $(x, y)$ of the decomposition problem for $a \neq 1$ is actually given by the first solution $y_{1}$, that is

$$
\begin{aligned}
& y=y_{1}=\frac{b}{1-a} \in[0,1) \\
& x=\frac{a}{1-y+a y}=\frac{a}{1-b} \in[0,1)
\end{aligned}
$$

The analysis of the case $a=1$ corresponding to the dogmatic BBA given by $m(A)=a=1, m(\bar{A})=b=0, m(A \cup \bar{A})=1-a-b=0$ shows that this BBA is not canonically decomposable by Dempster's rule. Why? Because one has to solve with $0 \leq x, y \leq 1$ and $1-x y \neq 0$ the system of equations $(x-x y) /(1-x y)=1$ and $(y-x y)(1-x y)=0$ which is satisfied for $x=1$ and $y \in[0,1)$, that is any value in $[0,1)$ can be chosen for $y$. Similarly, for the case $(a, b)=(0,1)$ one has to solve with $0 \leq x, y \leq 1$ and $1-x y \neq 0$ the system of equations $(x-x y) /(1-x y)=0$ and $(y-x y) /(1-x y)=1$ which is satisfied for $y=1$ and $x \in[0,1)$, that is any $x$ value in $[0,1)$ can be chosen. Therefore one sees that for the case $(a, b)=(1,0)$ and the case $(a, b)=(0,1)$ there is no unique decomposition of these dogmatic BBAs from Dempster's rule of combination. More generally, any dogmatic BBA $m(A)=a, m(\bar{A})=b$ with $a+b=1$ is not decomposable from Dempster's rule of combination for the case when $(a, b) \neq(1,0)$ and $(a, b) \neq(0,1)$ - See Theorem 4 with its proof in [6].

In summary, the canonical decomposition based on Dempster's rule of combination is possible only for nondogmatic BBA with $0<a<1,0<b<1$ and $a+b<1$ and we have $x=\frac{a}{1-b}$ and $y=\frac{b}{1-a}$. Dempster's rule does not

\footnotetext{
${ }^{9}$ Otherwise the denominator of (10) and (11) will equal zero.
} 
allow to obtain a canonical decomposition if the BBA is a Bayesian (dogmatic) dichotomous BBA.

\section{Example where Dempster's canonical decomposition is possible}

Consider $m(A)=a=0.6, m(\bar{A})=b=0.2$ and $m(A \cup \bar{A})=1-a-b=0.2$. The solution $(x, y)$ of the decomposition of $m(\cdot)$ based on Dempster's rule is

$$
x^{\prime}=\frac{a}{1-b}=\frac{0.6}{1-0.2}=0.75 \quad \text { and } \quad y^{\prime}=\frac{b}{1-a}=\frac{0.2}{1-0.6}=0.50
$$

Therefore, the pro- and contra- evidential BBAs $m_{p}$ and $m_{c}$ are given by

$$
\begin{array}{ll}
m_{p}(A)=x=0.75, & m_{p}(A \cup \bar{A})=1-x=0.25 \\
m_{c}(\bar{A})=y=0.50, & m_{c}(A \cup \bar{A})=1-y=0.50
\end{array}
$$

It can be verified that $D S\left(m_{p}, m_{c}\right)=m$.

If we make the PCR5-based canonical decomposition, we will obtain in this example $x \approx 0.6861$ and $y \approx 0.3628$. Therefore, the pro- and contra- evidential BBAs $m_{p}$ and $m_{c}$ based on the PCR5-based canonical decomposition are

$$
\begin{array}{ll}
m_{p}(A)=x=0.6861, & m_{p}(A \cup \bar{A})=1-x=0.3139 \\
m_{c}(\bar{A})=y=0.3628, & m_{c}(A \cup \bar{A})=1-y=0.6372
\end{array}
$$

It can be verified that $\operatorname{PCR} 5\left(m_{p}, m_{c}\right)=m$.

In the case where Dempster's rule can be applied for making the canonical decomposition (that is when $a+b<1$ ) we see that the canonical values (parameters) $x$ and $y$ can be very different from those obtained with PCR5 rule as shown in the previous example. This is normal because the principles of conflicting information redistribution of Dempster's rule and PCR5 rule are very different, and there is no link between parameters $x$ and $y$ obtained with Dempster's rule versus those obtained from PCR5. In PCR5 rule the conflict is a refined conflict, i.e. the conflict is split into partial conflicts, so in PCR5 the total conflict is more accurately redistributed than in Dempster's rule because each partial conflict is redistributed only to the elements involved into it, while in Dempster's rule the total conflict is redistributed to all focal elements, therefore even the elements that were not involved in the conflict receive conflicting mass, which is inaccurate.

It is worth noting that the internal conflict of $m$ based on Dempster's rule will be in this example $x y=0.75 \cdot 0.5=0.375$, whereas the internal conflict of $m$ based on PCR5 rule will be only $x y \approx 0.6861 \cdot 0.3628 \approx 0.2489$. In fact we can attest that the internal conflict obtained from PCR5-based canonical decomposition is always lesser (or equal) to the internal conflict obtained from Dempster-based canonical decomposition. Although such claim cannot be proved algebraically ${ }^{10}$,

${ }^{10}$ Because there is no simple analytical expressions for solutions $x$ and $y$ of PCR5-based canonical decomposition. 
we can always make a fine sampling of $(a, b)$ values in [0,1) satisfying $a+b<1$ to evaluate numerically $x$ and $y$ and compare the internal conflict $x y$ to the internal conflict, denoted $x^{\prime} y^{\prime}=\frac{a}{1-b} \cdot \frac{b}{1-a}$, obtained with Dempster-based canonical decomposition. In doing this we see that the difference $\Delta=x^{\prime} y^{\prime}-x y$ is always greater (or equal) to zero as clearly shown in figure 1 . This means that the PCR5based canonical decomposition is more efficient than Dempster-based canonical decomposition because it always yield pro- and contra-evidences which are less conflicting when using PCR5 rule than when using Dempster's rule, which is normal.

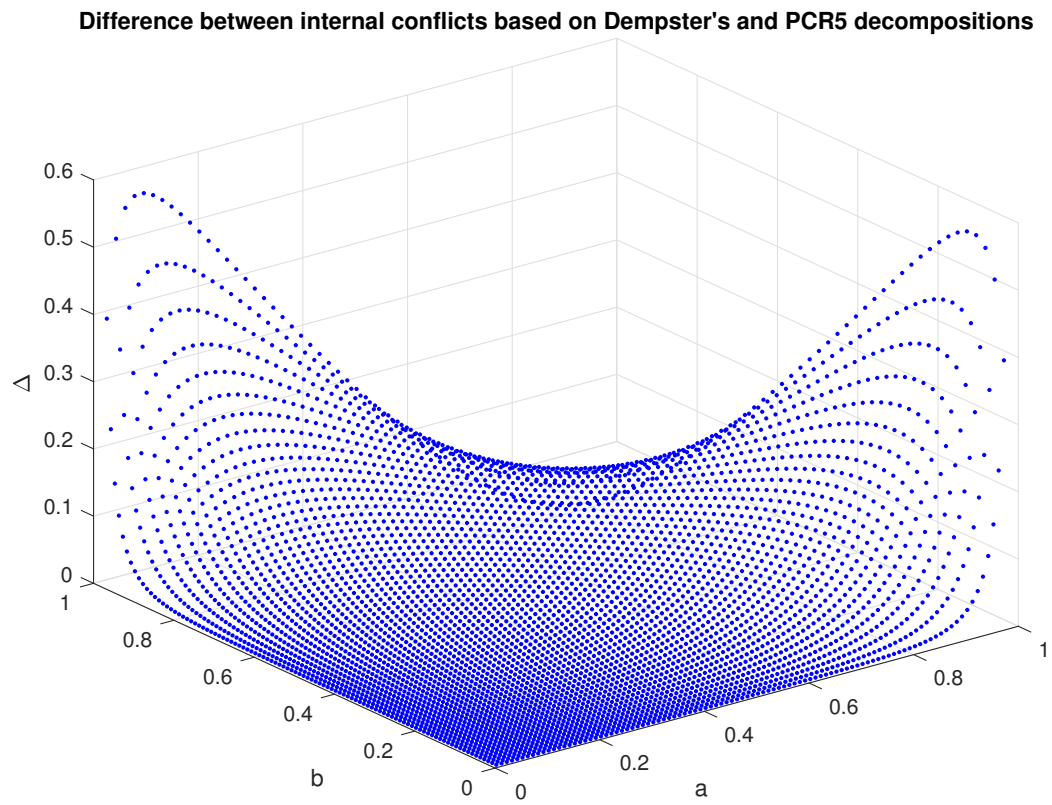

Fig. 1. Plot of $\Delta=x^{\prime} y^{\prime}-x y$ as function of $a$ and $b$.

It is important to keep in mind that Dempster-based canonical decomposition is only possible for non-dogmatic BBAs (when $a+b<1$ ) but cannot be obtained with dogmatic BBAs, whereas PCR5-based canonical decomposition works for all types of dichotomous BBAs (dogmatic and non-dogmatic ones).

\subsection{Simple example of PCR5-based canonical decomposition}

Let consider $m(A)=0.3, m(\bar{A})=0.4$ and $m(A \cup \bar{A})=1-m(A)-m(\bar{A})=0.3$, therefore $a=0.3$ and $b=0.4$. The quartic equation (9) becomes

$$
x^{4}-2.3 x^{3}+x^{2}+0.42 x-0.21=0
$$

The four solutions of this quartic equation are approximately ${ }^{11}$ 


$$
x_{1} \approx 1.5203, \quad x_{2} \approx-0.4243, \quad x_{3} \approx 0.7942, \quad x_{4} \approx 0.4099
$$

One sees that $x_{1}$ and $x_{2}$ are not acceptable solutions because they do not belong to $[0,1]$. If we take $x_{3} \approx 0.7942$ then will get $y_{3}=\left(a+b-x_{3}\right) /(1-$ $\left.x_{3}\right)=\left(0.7-x_{3}\right) /\left(1-x_{3}\right) \approx-0.4576$. We see that $y_{3} \notin[0,1]$ and therefore the pair $\left(x_{3}, y_{3}\right)$ cannot be a solution of the PCR5-based canonical decomposition problem for the BBA $m(\cdot)$ of this example. If we take $x_{4} \approx 0.4099$ then will get $y_{4}=\left(a+b-x_{4}\right) /\left(1-x_{4}\right)=\left(0.7-x_{4}\right) /\left(1-x_{4}\right) \approx 0.4916$ which belongs to $[0,1]$. So the pair $\left(x_{4}, y_{4}\right) \in[0,1]^{2}$ is the unique solution of the canonical decomposition problem. Therefore the canonical masses $m_{p}(\cdot)$ and $m_{c}(\cdot)$ are given by

$m_{p}(A) \approx 0.4099, \quad m_{p}(A \cup \bar{A}) \approx 0.5901 \quad$ and $\quad m_{c}(\bar{A}) \approx 0.4916, \quad m_{c}(A \cup \bar{A}) \approx 0.5084$

It can be verified that $\operatorname{PCR} 5\left(m_{p}, m_{c}\right)=m$.

\subsection{Advantages and limitation of PCR5-based decomposition}

The PCR5-based canonical decomposition offers the following advantages:

1. It is well justified theoretically.

2. It gives us access to the simpler pro- and contra-evidences $m_{p}(\cdot)$ and $m_{c}(\cdot)$ which are unique and always exist for any possible (dogmatic, or non-dogmatic) dichotomous BBA $m(\cdot)$.

3. It allows to define clearly the notion of internal conflict of a dichotomous source of evidence simply as $K_{\text {int }}(m) \triangleq m_{p}(A) m_{c}(\bar{A})$.

4. It always provides less conflicting pro- and contra-evidences than what we would obtain with Dempster's rule when considering non-dogmatic dichotomous BBA $m(\cdot)$. This proves the superiority of PCR5-based canonical decomposition over Dempster's-based canonical decomposition in general.

5. It allows also to adjust or revise ${ }^{12}$ quite easily a dichotomous source of evidence (if needed) according to the knowledge one has on it by reinforcing or discounting its pro- or contra-evidential BBA.

6. It can be easily achieved with classical numerical solvers on the shelf.

7. The decomposition can be done off-line for many sampled $(a, b)$ values at any precision we want, and stored in computer memory for working directly with $m_{p}(\cdot)$ and $m_{c}(\cdot)$ instead of making the decomposition on the fly. This is of prime importance for real-time applications where this method could be used.

8. It allows to establish efficient fast ${ }^{13}$ suboptimal PCR5 fusion scheme, see [10] for details, examples and evaluations.

The only important limitation of this PCR5-based canonical decomposition is that it applies only to dichotomous BBAs, and it seems very difficult (maybe impossible) to use or to extend it for making directly some new canonical decomposition of non dichotomous BBAs. Because of this limitation the use of

\footnotetext{
11 The solutions can be easily obtained with the roots command of Matlab ${ }^{\mathrm{TM}}$.

12 This point is not detailed here because is out of the scope of this paper.

${ }^{13}$ Where the complexity is linear with the number of dichotomous BBAs to fuse.
} 
PCR5-based canonical decomposition appears, at first glance, quite restrictive for being really useful in applications involving non dichotomous BBAs. Of course in applications working with dichotomous BBAs (like those in robotics or for autonomous vehicle navigation using belief-based perception based on grid occupancy) this PCR5-based canonical decomposition may have a great interest. In fact we have already used it for belief-based inter-criteria analysis in [11] and that is why we do not present our results in this work. Nevertheless we will show in the next section how this PCR5-based canonical decomposition could be used for the decision-making support in a more general context involving many non-dichotomous BBAs. This is a problem which has not been addressed in [6].

\section{Decision-making using PCR5-based decomposition}

In this section we propose a new simple general decison-making scheme based on PCR5-based canonical decomposition of dichotomous BBA. We consider $S>2$ distinct sources of evidence characterized by their $\mathrm{BBAs}^{14} m_{s}^{\Theta}(\cdot)$ defined over the same (possibly non dichotomous) FoD $\Theta=\left\{\theta_{1}, \ldots, \theta_{n}\right\}$, with $n>1$.

Can we exploit the PCR5-based canonical decomposition in this context to make a decision? How? We answer positively to the first question and explain in details how we can proceed. For this, we need to express the problem in the framework of dichotomous BBAs that has been presented in the previous section. More precisely, suppose one has a BBA $m^{\Theta}$ (.) defined on $2^{\Theta}$ with $|\Theta| \geq 2$, then based on Bel and Pl formulas (1)-(2), it is always possible to calculate $B e l^{\Theta}(X)$ and $P l^{\Theta}(X)$ for any $X \in 2^{\Theta}$. From $\mathrm{Bel}^{\Theta}(X)$ and $P l^{\Theta}(X)$ one can always build a simpler coarsened dichotomous BBA on the dichotomous (coarsened) FoD $\Theta_{X} \triangleq\{X, \bar{X}\}$ if $X \neq \emptyset$ and $X \neq \Theta_{X}$ as follows

$$
\begin{aligned}
& m^{\Theta_{X}}(X)=\operatorname{Bel}^{\Theta}(X) \\
& m^{\Theta_{X}}(\bar{X})=1-P l^{\Theta}(X) \\
& m^{\Theta_{X}}(X \cup \bar{X})=P l^{\Theta}(X)-B e l^{\Theta}(X)
\end{aligned}
$$

Hence, $\operatorname{Bel}^{\Theta_{X}}(X)=m^{\Theta_{X}}(X)=\operatorname{Bel}(X)$ and $P l^{\Theta_{X}}(X)=m^{\Theta_{X}}(X)+m^{\Theta_{X}}(X \cup$ $\bar{X})=B e l^{\Theta}(X)+P l^{\Theta}(X)-B e l^{\Theta}(X)=P l^{\Theta}(X)$. This dichotomous BBA $m^{\Theta_{X}}(\cdot)$ can always be decomposed canonically into its pro- and contra-evidences $m_{p}^{\Theta_{X}}($. and $m_{c}^{\Theta_{X}}($.$) .$

Therefore, instead of combining $S>1$ non dichotomous BBAs $m_{s}^{\Theta}($.$) for$ $s=1,2, \ldots, S$ altogether from which a decision is classically drawn, we propose to make the decision from the set of all combined coarsened BBAs relatively to each possible dichotomous frame of discernment $\Theta_{X}$. Of course this decisionscheme is only suboptimal because the whole information is not processed (combined) altogether, but separately using only the coarsened (less informative) BBAs $m_{s}^{\Theta_{X}}(X)$. However, this method allows to use fast suboptimal PCR5 fusion of $m_{s}^{\Theta_{X}}(X)$ thanks to PCR5-based canonical decomposition as presented in

\footnotetext{
${ }^{14}$ For clarity, we need to introduce in the notations a superscript to indicate the FoD
} we are working on. 
[10] which can be applied with many (hundreds or even thousands) sources of dichotomous BBAs. With this simple suboptimal decision-scheme we can easily restrict the domain $\mathcal{D}$ on which the decisions can be made, for instance $\mathcal{D}$ can be chosen as the set of singletons of $2^{\Theta}$, or any other subset of $2^{\Theta}$ depending on the application under concern as it will be shown in the next section. The generic steps of the method we propose are as follows:

- Inputs: BBAs $m_{s}^{\Theta}(\cdot), s=1, \ldots, S$, and the decision domain $\mathcal{D} \subset 2^{\Theta}$.

- Step 1: For $s=1, \ldots, S$, coarsening of $m_{s}^{\Theta}(\cdot)$ into dichotomous BBA $m_{s}^{\Theta_{X}}(\cdot)$, for each $X \in \mathcal{D}$ based on (17)-(19).

- Step 2: For $s=1, \ldots, S$, PCR5-based canonical decomposition of $m_{s}^{\Theta_{X}}(\cdot)$ to get pro- and contra-evidences $m_{p, s}^{\Theta_{X}}(\cdot)$ and $m_{c, s}^{\Theta_{X}}(\cdot)$.

- Step 3: Conjunctive fusion of all the pro-evidences $m_{p, s}^{\Theta_{X}}(\cdot)$ to get $m_{p}^{\Theta_{X}}(\cdot)$.

- Step 4: Conjunctive fusion of all the contra-evidences $m_{c, s}^{\Theta_{X}}(\cdot)$ to get $m_{c}^{\Theta_{X}}(\cdot)$.

- Step 5: PCR5 fusion of $m_{p}^{\Theta_{X}}(\cdot)$ with $m_{c}^{\Theta_{X}}(\cdot)$ to get $m_{P C R 5}^{\Theta_{X}}(\cdot)$ for $X \in \mathcal{D}$.

- Step 6: Decision-making from the set of the combined coarsened dichotomous BBAs $\left\{m_{P C R 5}^{\Theta_{X}}(\cdot), X \in \mathcal{D}\right\}$ to get the final decision $\hat{X} \in \mathcal{D}$.

- Output: the final decision $\hat{X} \in \mathcal{D}$

In steps 3 and 4 we use the conjunctive fusion because there is no conflict between all pro-evidences $m_{p, s}^{\Theta_{X}}(\cdot)$, and there is also no conflict between all contra-evidences $m_{c, s}^{\Theta_{X}}(\cdot), s=1, \ldots, S$. The steps 1 to 5 do not require high computational burden and they can be done very quickly, specially if PCR5-based decompositions have been done off-line (as they should be) [10].

We must detail a bit more the principle of the decision-making for the step 6. Actually, the decision-making for step 6 can be interpreted as a decisionmaking problem from a set or coarsened BBAs $m_{P C R 5}^{\Theta X}(\cdot)$ defined over different dichotomous FoD $\Theta_{X}$ which are all the different coarsenings of the whole (refined original) FoD $\Theta$. In this paper we propose two methods to make the decision from the set of coarsened BBAs $\left\{m_{P C R 5}^{\Theta_{X}}(\cdot), X \in \mathcal{D}\right\}$.

\subsection{Method 1 for Step 6}

This method is very simple. We take the decision $\hat{X}$ corresponding to the largest value of $m_{P C R 5}^{\Theta_{X}}(X)$, that is

$$
\hat{X}=\arg \max _{X \in \mathcal{D}}\left(m_{P C R 5}^{\Theta_{X}}(X)\right)
$$

If there exist several arguments having the largest value (i.e. there is a tie), we select the one whose $m_{P C R 5}^{\Theta_{X}}(\bar{X})$ is smaller.

Example 1 (without tie): Suppose $\Theta=\{A, B, C, D, E\}$ and we want to make a decision/choice only among the elements of $\mathcal{D}=\{A, B, C\}$. Suppose after applying steps 1-5 we get the following $3 \mathrm{BBAs}$

$$
\begin{array}{lll}
m_{P C R 5}^{\Theta_{A}}(A)=0.3, & m_{P C R 5}^{\Theta_{A}}(\bar{A})=0.2, & m_{P C R 5}^{\Theta_{A}}(A \cup \bar{A})=0.5 \\
m_{P C R 5}^{\Theta_{B}}(B)=0.1, & m_{P C R 5}^{\Theta_{B}}(\bar{B})=0.5, & m_{P C R 5}^{\Theta_{B}}(B \cup \bar{B})=0.4 \\
m_{P C R 5}^{\Theta_{C}}(C)=0.4, & m_{P C R 5}^{\Theta_{C}}(\bar{C})=0.3, & m_{P C R 5}^{\Theta_{C}}(C \cup \bar{C})=0.3
\end{array}
$$


The decision will be $\hat{X}=C$ because $m_{P C R 5}^{\Theta_{C}}(C)>m_{P C R 5}^{\Theta_{A}}(A)>m_{P C R 5}^{\Theta_{B}}(B)$.

Example 2 (with tie) We consider same $m_{P C R 5}^{\Theta_{B}}($.$) and m_{P C R 5}^{\Theta_{C}}($.$) as in example$ 1 but $m_{P C R 5}^{\Theta_{A}}($.$) is given by m_{P C R 5}^{\Theta_{A}}(A)=0.4, m_{P C R 5}^{\Theta_{A}}(\bar{A})=0.2$, and $m_{P C R 5}^{\Theta_{A}}(A \cup$ $\bar{A})=0.4$. In this case, there is a tie between $A$ and $C$ because $m_{P C R 5}^{\Theta_{A}}(A)=$ $m_{P C R 5}^{\Theta_{C}}(C)=0.4$. But because $m_{P C R 5}^{\Theta_{A}}(\bar{A})<m_{P C R 5}^{\Theta_{C}}(\bar{C})$ we will take $\hat{X}=A$ as the final decision.

The interest of this method is above all its simplicity, but it does not allow to quantify the quality (trustfulness) of the decision which is often useful and required in decision-making support systems, and that is why we propose a second method for the decision-making of step 6 .

\subsection{Method 2 for Step 6}

This second method is a bit more sophisticate but it circumvents the exponential complexity of the direct PCR6 fusion of $S \geq 2$ BBAs defined on non dichotomous FoD $\Theta$. Once the step 5 is accomplished we propose to fuse altogether the (coarsened) dichotomous $m_{P C R 5}^{\Theta_{X}}(\cdot)$ and to apply the decision-making method based on the distance between the belief intervals [12]. Because the fusion must operate on the same common frame, we need just to express each BBA $m_{P C R 5}^{\Theta_{X}}(\cdot)$ as a dichotomous BBA on $\Theta$ which is denoted $m_{P C R 5}^{\Theta_{X} \uparrow \Theta}(\cdot)$. This is done very easily by just expressing each $\bar{X}$ as the disjunction of all elements of $\Theta$ included in $\bar{X}$. The fusion of BBAs $m_{P C R 5}^{\Theta x \uparrow \Theta}(\cdot)$ is done by the weighted averaging rule of combination, where each weighting factor depends on the decisioning-making easiness of the BBA $m_{P C R 5}^{\Theta_{X}}(\cdot)$ to fuse. The easier the decision-making, the higher the weighting factor. We summarize this method 2:

1) For each $X \in \mathcal{D}$, establish $m_{P C R 5}^{\Theta_{X} \uparrow}(\cdot)$ from $m_{P C R 5}^{\Theta_{X}}(\cdot)$

2) For each $X \in \mathcal{D}$, compute the weighting factor $w(X)$ of $m_{P C R 5}^{\Theta_{X} \uparrow \Theta}(\cdot)$ by

$$
w(X)=\frac{1}{C}\left(1-h\left(m_{P C R 5}^{\Theta_{X} \uparrow \Theta}\right)\right)
$$

where $C=\sum_{X \in \mathcal{D}}\left(1-h\left(m_{P C R 5}^{\Theta_{X} \uparrow \Theta}\right)\right)$ is a normalization factor, and where $h\left(m_{P C R 5}^{\Theta_{X} \uparrow \Theta}\right)=H\left(m_{P C R 5}^{\Theta_{X} \uparrow \Theta}\right) / H_{\max } \in[0,1]$ is the normalized pignistic entropy of the BBA $m_{P C R 5}^{\Theta_{X} \uparrow \Theta}$ defined by $H\left(m_{P C R 5}^{\Theta_{X} \uparrow \Theta}\right)=-\sum_{X \in 2}{ }^{\Theta} \operatorname{Bet} P(X) \log _{2}(\operatorname{Bet} P(X))$ and $\operatorname{Bet} P(X)$ is the pignistic probability of $X$ [13], and $H_{\max }=\log _{2}|\Theta|$.

3) Make the weighting average of $m_{P C R 5}^{\Theta_{X} \uparrow \Theta}(\cdot)$ for all $X \in \mathcal{D}$ to get the BBA

$$
m^{\Theta}(\cdot)=\sum_{X \in \mathcal{D}} w(X) m_{P C R 5}^{\Theta X \uparrow}(\cdot)
$$

4) From $m^{\Theta}(\cdot)$ make the decision based on minimum of belief-interval distance $[12]$, that is

$$
\hat{X}=\arg \min _{X \in \mathcal{D}} d_{B I}\left(m^{\Theta}, m_{X}^{\Theta}\right)
$$


where $m_{X}^{\Theta}$ is the BBA focused on $X$ that is $m_{X}^{\Theta}(X)=1$ and $m_{X}^{\Theta}(Y)=0$ if $Y \neq X$, and where $d_{B I}(.,$.$) is the belief-interval distance defined by (see$ [12] for details, justification and examples)

$$
d_{B I}\left(m_{1}, m_{2}\right) \triangleq \sqrt{N_{c} \cdot \sum_{X \in 2^{\Theta}} d_{W}^{2}\left(B I_{1}(X), B I_{2}(X)\right)}
$$

where $N_{c}=1 / 2^{|\Theta|-1}$ is a normalization factor to have $d_{B I}\left(m_{1}, m_{2}\right) \in[0,1]$, and $d_{W}\left(B I_{1}(X), B I_{2}(X)\right)$ is the Wassertein's distance between belief intervals $B I_{1}(X) \triangleq\left[B e l_{1}(X), P l_{1}(X)\right]=\left[a_{1}, b_{1}\right]$ and $B I_{2}(X) \triangleq\left[B e l_{2}(X), P l_{2}(X)\right]=$ $\left[a_{2}, b_{2}\right]$ given by

$$
d_{W}\left(\left[a_{1}, b_{1}\right],\left[a_{2}, b_{2}\right]\right) \triangleq \sqrt{\left[\frac{a_{1}+b_{1}}{2}-\frac{a_{2}+b_{2}}{2}\right]^{2}+\frac{1}{3}\left[\frac{b_{1}-a_{1}}{2}-\frac{b_{2}-a_{2}}{2}\right]^{2}}
$$

5) The quality (or trustfulness) of the decision is given by

$$
q(\hat{X}) \triangleq 1-\frac{d_{B I}\left(m, m_{\hat{X}}\right)}{\sum_{X \in \mathcal{D}} d_{B I}\left(m, m_{X}\right)}
$$

$q(\hat{X}) \in[0,1]$ becomes maximum (equal to one) when $d_{B I}\left(m^{\Theta}, m_{\hat{X}}^{\Theta}\right)$ is zero, which means that $m^{\Theta}(\cdot)$ is focused only on $\hat{X}$. The higher $q(\hat{X})$ is, the more confident in the decision $\hat{X}$ we are. When there exists a tie between multiple decisions $\left\{\hat{X}_{j}, j>1\right\}$, then the prudent decision corresponding to their disjunction $\hat{X}=\cup_{j} \hat{X}_{j}$ should be preferred (if allowed), or we can apply the method 1 to resolve the tie, or in desperation select randomly $\hat{X}$ among the elements $\hat{X}_{j}$ involved in the tie.

Of course we could adopt a more complicate method where the averaging fusion could operate on all the possible dichotomous BBAs related with each element $X \in 2^{\Theta \backslash\{\emptyset, \Theta\}}$ instead of $X \in \mathcal{D}$, but this would substantially increase the computational burden. Because the decision $\hat{X}$ must be constrained to belong to $\mathcal{D}$, we restrict the fusion to be applied only for the dichotomous BBAs related to these elements only. By doing this we can reduce substantially the computational burden if $|\mathcal{D}|$ is much lesser than $2^{|\Theta|}$.

For convenience, we show how works the method 2 in the previous Example 1 using the same $\Theta$ and $\mathcal{D}=\{A, B, C\}$. We have to make the weighted average of the three following BBAs

$$
\begin{array}{lll}
m_{P C R 5}^{\Theta_{A} \uparrow \Theta}(A)=0.3, & m_{P C R 5}^{\Theta_{A} \uparrow \Theta}(B \cup C \cup D \cup E)=0.2, & m_{P C R 5}^{\Theta_{A} \uparrow \Theta}(A \cup \bar{A}=\Theta)=0.5 \\
m_{P C R 5}^{\Theta_{B} \uparrow \Theta}(B)=0.1, & m_{P C R 5}^{\Theta_{B} \uparrow \Theta}(A \cup C \cup D \cup E)=0.5, & m_{P C R 5}^{\Theta_{B} \uparrow \Theta}(B \cup \bar{B}=\Theta)=0.4 \\
m_{P C R 5}^{\Theta_{C} \uparrow \Theta}(C)=0.4, & m_{P C R 5}^{\Theta_{C} \uparrow \Theta}(A \cup B \cup D \cup E)=0.3, & m_{P C R 5}^{\Theta_{C} \uparrow \Theta}(C \cup \bar{C}=\Theta)=0.3
\end{array}
$$

with $B \cup C \cup D \cup E=\bar{A}, A \cup C \cup D \cup E=\bar{B}$ and $A \cup B \cup D \cup E=\bar{C}$. The pignistic entropies are respectively equal to $H\left(m_{P C R 5}^{\Theta_{A} \uparrow \Theta}\right) \approx 2.1710, H\left(m_{P C R 5}^{\Theta_{B} \uparrow \Theta}\right) \approx 2.3201$ and $H\left(m_{P C R 5}^{\Theta_{C} \uparrow}\right) \approx 2.0754$, and their normalized values are $h(A) \approx 2.1710 / 2.3219=$ 
$0.9350, h(B) \approx 2.3201 / 2.3219=0.9992$ and $h(C) \approx 2.0754 / 2.3219=0.8938$. From Eq. $(21)$ we get the weighting factors $w(A) \approx 0.37803, w(B) \approx 0.00463$ and $w(C) \approx 0.61734$, and the weighted average BBA is

$$
\begin{aligned}
& m^{\Theta}(A)=w(A) m_{P C R 5}^{\Theta_{A} \uparrow \Theta}(A)+w(B) \cdot 0+w(C) \cdot 0 \approx 0.1134 \\
& m^{\Theta}(B)=w(A) \cdot 0+w(B) m_{P C R 5}^{\Theta_{B} \uparrow \Theta}(B)+w(C) \cdot 0 \approx 0.0005 \\
& m^{\Theta}(C)=w(A) \cdot 0+w(B) \cdot 0+w(C) m_{P C R 5}^{\Theta_{C} \uparrow \Theta}(C) \approx 0.2469 \\
& m^{\Theta}(B \cup C \cup D \cup E)=w(A) m_{P C R 5}^{\Theta_{A} \uparrow \Theta}(B \cup C \cup D \cup E)+w(B) \cdot 0+w(C) \cdot 0 \approx 0.0756 \\
& m^{\Theta}(A \cup C \cup D \cup E)=w(A) \cdot 0+w(B) m_{P C R 5}^{\Theta_{B} \uparrow \Theta}(A \cup C \cup D \cup E)+w(C) \cdot 0 \approx 0.0023 \\
& m^{\Theta}(A \cup B \cup D \cup E)=w(A) \cdot 0+w(B) \cdot 0+w(C) m_{P C R 5}^{\Theta_{C} \uparrow \Theta^{-}}(A \cup B \cup D \cup E) \approx 0.1852 \\
& m^{\Theta}(\Theta)=w(A) m_{P C R 5}^{\Theta_{A} \uparrow \Theta}(\Theta)+w(B) m_{P C R 5}^{\Theta_{B} \uparrow \Theta}(\Theta)+w(C) m_{P C R 5}^{\Theta_{C} \uparrow \Theta}(\Theta)=0.3761
\end{aligned}
$$

From Eq. (24) we get $d_{B I}\left(m^{\Theta}, m_{A}^{\Theta}\right) \approx 0.6818, d_{B I}\left(m^{\Theta}, m_{B}^{\Theta}\right) \approx 0.7541$ and $d_{B I}\left(m^{\Theta}, m_{C}^{\Theta}\right) \approx 0.5874$ because $d_{B I}\left(m^{\Theta}, m_{C}^{\Theta}\right)<d_{B I}\left(m^{\Theta}, m_{A}^{\Theta}\right)<d_{B I}\left(m^{\Theta}, m_{B}^{\Theta}\right)$. Thus the final decision must be $\hat{X}=C$ because it corresponds to the smallest $d_{B I}$ distance value. This decision is the same as with method 1. Based on Eq. (25) one has $q(\hat{X}=C) \approx 0.7096$ indicating a pretty good trustful decision because it is much greater than 0.5 . If one have preferred $\hat{X}=A$ (the second best choice) then $q(\hat{X}=A) \approx 0.6630$ which is a bit worse, and for $\hat{X}=B$ one gets the least trustful decision because $q(\hat{X}=B) \approx 0.6273$. Note that a more optimistic attitude (if preferred) could be obtained by replacing the BetP probability by the DSmP probability [5] (Chap. 3 of Vol. 3) in the entropy derivation.

\section{Conclusions}

In this work we have presented a very new methodology for decision-making under uncertainty in the framework of belief functions thanks to the unique PCR5based canonical decomposition of any (dogmatic or non-dogmatic) dichotomous BBAs. We have shown that this new canonical decomposition provides less conflicting contra- and pro-evidences with respect to the decomposition based on Dempster's rule when the latter can be applied. Any BBAs defined on a general (non dichotomous) frame of discernment can be transformed into a set of coarsened dichotomous BBAs that can always be decomposed canonically and combined easily and quickly in one PCR5 fusion step to get a suboptimal fusion result for each element of the decision space under consideration. The final decision can be made in two ways: either by a simple comparative analysis of masses of elements of the decision space, or on the minimization of belief-interval distance which also offers the advantage of quantifying the quality of the decision. The evaluation of this new methodology for real applications is under progress and it will reported in forthcoming publications. 


\section{References}

1. Shafer G.: A Mathematical theory of evidence, Princeton University Press (1976)

2. Zadeh, L.A.: On the validity of Dempster's rule of combination. ERL Memo M79/24, Department of EECS, Univ. of California, Berkeley, U.S.A. (1979)

3. Zadeh, L.A.: A simple view of the Dempster-Shafer theory of evidence and its implication for the rule of combination. The Al Magazine, 7(2), 85-90 (1986).

4. Dezert J., Tchamova A.: On the validity of Dempster's fusion rule and its interpretation as a generalization of bayesian fusion rule. Int. J. of Intelligent Syst. 29(3), 223-252 (2014)

5. Smarandache, F., Dezert J. (Editors): Advances and applications of DSmT for information fusion, American Research Press, Vols. 1-4 (2004-2015)

6. Dezert, J., Smarandache, F.: Canonical decomposition of dichotomous basic belief assignment. International Journal of Intelligent Systems, 1-21 (2020)

7. Smets, P.: The canonical decomposition of a weighted belief. In: Proc. of Int. Joint Conf. on Artif. Intell., 1896-1901, San Mateo, CA, USA (1995)

8. Yager, R.: On the Dempster-Shafer framework and new combination rules. Information Sciences 41, 93-138 (1987)

9. Dubois, D., Prade, H.: Representation and combination of uncertainty with belief functions and possibility measures. Computational Intelligence 4, 244-264 (1988)

10. Dezert, J., Smarandache, F., Tchamova, A., Han, D.: Fast fusion of basic belief assignments defined on a dichotomous frame of discernment. In: Proc. of Fusion 2020, Pretoria, South Africa (2020)

11. Dezert, J., Fidanova, S., Tchamova, A.: Fast BF-ICrA method for the evaluation of MO-ACO algorithm for WSN layout. In: Proc. of FedCSIS Int. Conference, Sofia, Bulgaria (2020)

12. Han, D., Dezert, J., Yang, Y.: Belief interval based distances measures in the theory of belief functions. IEEE Trans. on SMC, 486, 833-850 (2018)

13. Smets, P., Kennes, R.: The transferable belief model. Art. Intell., 662, 191-234 (1994) 